\title{
Engineering spin exchange in nonbipartite graphene zigzag edges
}

\author{
R. Ortiz, ${ }^{1}$ J. L. Lado, ${ }^{2}$ M. Melle-Franco, ${ }^{3}$ and J. Fernández-Rossier ${ }^{1,2}$ \\ ${ }^{1}$ Departamento de Física Aplicada, Universidad de Alicante, 03690, Spain \\ ${ }^{2}$ International Iberian Nanotechnology Laboratory (INL), Av. Mestre José Veiga, 4715-330 Braga, Portugal \\ ${ }^{3}$ CICECO, Departamento de Química, Universidade de Aveiro, 3810-193 Aveiro, Portugal
}

(Received 15 June 2016; revised manuscript received 17 August 2016; published 13 September 2016)

\begin{abstract}
The rules that govern spin exchange interaction in pristine graphene nanostructures are constrained by the bipartite character of the lattice, so that the sign of the exchange is determined by whether magnetic moments are on the same sublattice or not. The synthesis of graphene ribbons with perfect zigzag edges and a fluoranthene group with a pentagon ring, a defect that breaks the bipartite nature of the honeycomb lattice, has been recently demonstrated. Here we address how the electronic and spin properties of these structures are modified by such defects, both for indirect exchange interactions as well as the emergent edge magnetism, studied both with density functional theory and mean-field Hubbard model calculations. In all instances we find that the local breakdown of the bipartite nature at the defect reverts the sign of the otherwise ferromagnetic correlations along the edge, introducing a locally antiferromagnetic intraedge coupling and, for narrow ribbons, also revert the antiferromagnetic interedge interactions that are normally found in pristine ribbons. Our findings show that these pentagon defects are a resource that permits us to engineer the spin exchange interactions in graphene-based nanostructures.
\end{abstract}

DOI: 10.1103/PhysRevB.94.094414

A central concept in the vast field of carbon-based nanostructures is the fact that their electronic properties can change dramatically depending on their atomic structure. Thus, graphite, graphene, nanotubes, and fullerenes all share the same atomic scale building blocks, carbon atoms with $s p^{2}$ chemical bond, yet their electronic properties are very different [1]. Many remarkable electronic properties of graphene and other $s p^{2}$ nanostructures, such as electron-hole symmetry [2-4], the existence of zero-energy modes [2,5], and the rules that govern spin exchange interactions [6-9] derive from the bipartite nature of the honeycomb lattice.

A bipartite lattice can be split in two interpenetrating sublattices, $A$ and $B$, such that first neighbors of $A$ sites are always $B$ sites, and vice versa. Whereas in two-dimensional (2D) graphene the wave functions have the same weight on both sublattices, in structures where there are more atoms of one type than the other, such as zigzag edges, there are zero modes whose wave function is $100 \%$ sublattice polarized $[2,8]$. These states play a crucial role in our understanding of one of the most exciting theory predictions regarding graphene so far, namely, the existence of local moments with ferromagnetic correlations in sublattice imbalanced graphene structures, such as zigzag edges $[7,8,10-13]$, graphene functionalized with hydrogen [13-15], and a variety of planar aromatic hydrocarbons $[16,17]$. Whereas a direct experimental local probe of the magnetization is still missing, indirect experimental evidence in full agreement with density functional theory (DFT) and model Hamiltonian calculations [18-21] supports the existence of sublattice polarized states that most likely host unpaired electrons.

Interestingly, the chemical approach recently reported by Ruffieux et al. $[19,20]$ has produced both ribbons with large sections of pristine zigzag edges as well as edges decorated with a fluoranthene group (FG), ${ }^{1}$ as those shown in Figs. 1(a)

\footnotetext{
${ }^{1}$ The FG is defined by analogy with the fluoranthene molecule, which structurally comprises one napthalene group and one benzene
}

and 3(a), that break the bipartite character of the lattice, on account of the presence of a pentagon at the edge. This naturally leads to the question that we address in this work: what is the fate of edge states, and the spin interactions they produce, in the case of zigzag ribbons decorated with nonbipartite intrusions. Previous work had addressed the magnetism of an individual octagon-pentagon pair in bulk graphene [22] (away from the edge), the magnetic properties of a line of pentagon-pentagon-octagon (558) defects, as a grain boundary in graphene [23], but the properties of the recently found [20] zigzag ribbons decorated with FGs have remained unexplored. To address the question, we consider both indirect exchange interactions between some extrinsic spins, mediated by the electrons in the FG decorated ribbons, as well as the edge magnetism that emerges, according to our Hubbard model, treated within the noncollinear mean-field approximation $[24,25]$ as well as calculations based on density functional theory (DFT).

Our main findings are the following. First, the presence of these defects locally depletes both the edge states and the edge magnetization, but edge magnetism with ferromagnetic correlations persists at the pristine sections as long as the FG groups in the edge are not too close to each other. Second, and more important, the exchange interactions of two zigzag segments separated by a single FG are antiferromagnetic, whereas the face-to-face spin correlations can become ferromagnetic, both results at odds with the case of pristine ribbons $[7,10,11]$.

In order to study the effect of the edges decorated with FG, we consider finite-size graphene islands (see Fig. 1). We first study them with the standard tight-binding model with one orbital and first-neighbor hopping $t$. As we show below, our DFT results indicate that the presence of the FG

group joined together by a pentagonal ring. In our case, the napthalene group is replaced by the graphene ribbon, so that the pentagonal rings covalently link zigzag graphene edges with benzene rings. [See Fig. 1(a)]. 


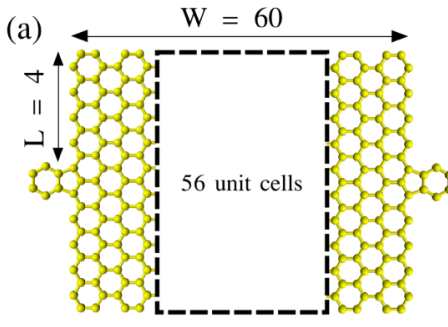

(c)

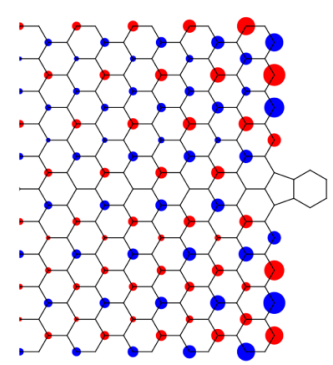

(d)
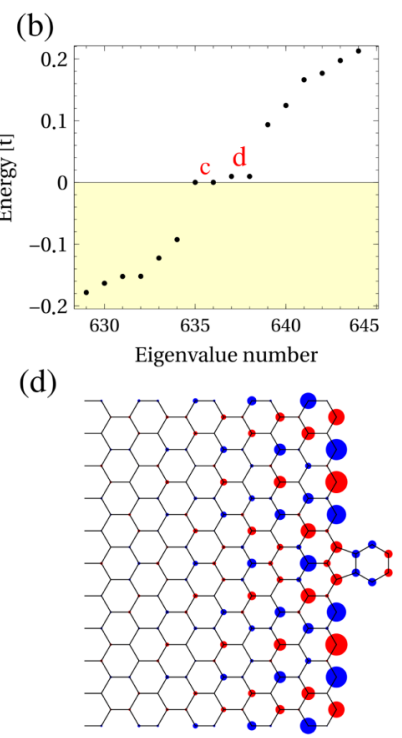

FIG. 1. (a) Large graphene island with zigzag edges functionalized with a FG. (b) Energy spectrum, for eigenvalues close to the Fermi energy, showing four in-gap states, two per right/left edge. The twofold degeneracy in the smallest eigenvalues corresponds to the two lateral edges. (c), (d) are the wave functions labeled in (b), showing the edge nature of the in-gap states, in particular their bonding and antibonding character. Color in (c), (d) labels the sign, whereas the size indicates the magnitude of the component. The splitting between the two states in the edge allows us to map the system into a two-site tight-binding model, which is expected to develop antiferromagnetic correlations. The width of the island was chosen so that the bulk is gapped. The dimensions of the island are shown in (a), width of 60 unit cells and four carbon atoms per semi-edge.

preserves the planar geometry of the system, so that the $\pi$ orbitals are still decoupled from the $\sigma$ orbitals, validating this model. For simplicity, we assume the same hopping integral $t_{\mathrm{C}-\mathrm{C}}=-\mathrm{t}=-2.7 \mathrm{eV}$ among all first-neighbor bonds, including also those of the defect. In order to understand the properties of an individual edge, we compute the spectrum of a rectangular-shaped graphene island, terminated with two short zigzag edges with $L$ edge atoms each, separated by a distance $W$. We choose $L=10$ hexagons so that the bulk spectrum is gapped. For the pristine ribbon there are three in-gap $E \simeq 0$ states whose wave functions are localized at each edge, and their wave function is sublattice polarized (not shown). The addition of a single FG at each side breaks the bipartite character of the lattice and has the following consequences. First, the number of localized in-gap edge states is reduced from six (three per edge) to four (two per edge). Second, the remaining edge states are split in energy, so that only two of them have $E \simeq 0$, the other two move upwards in energy. This breaks electron-hole symmetry. ${ }^{2}$ The intraedge splitting arises from the formation of a bonding nonbonding pair of two modes that are localized at both sides of the defect. In the experiment of Ruffieux et al. [20], the distance between zigzag edges was

\footnotetext{
${ }^{2}$ For this reason, the choice of sign of $t$ matters, in contrast to the case of bipartite systems.
}

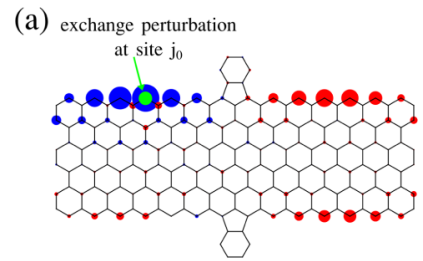

(c)

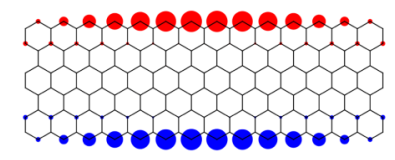

(b)

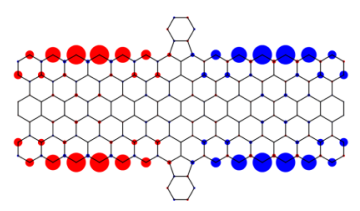

(d)

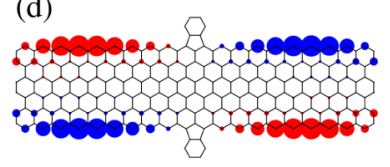

FIG. 2. (a) Map of the nonlocal spin susceptibility $\chi_{i j_{0}}$, when $j_{0}$ is fixed at the green atom at the top-left edge, and $i$ runs over the entire island. The color reflects the sign of $\chi_{i j_{0}}$, and the size the magnitude. (b), (c), (d) show the spatial profile of the eigenstate $v$ of $\chi_{i j}$, so that $\chi_{i j} v_{i}=\lambda v_{j}$, with $\lambda$ the largest eigenvalue, that characterizes the magnetic density right below the critical temperature. The color represents the sign of the component $v_{i}$, and the size its magnitude. (c) corresponds to the ribbon without FG, showing conventional intraedge ferromagnetic and interedge antiferromagnetic correlations. (b), (d) show the antiferromagnetic correlations in the same edge due to the FG group, while the interedge correlations depend on the size of the island (b), (d).

$W=5$ hexagons, so that hybridization between states at both edges takes place.

We now address the spin exchange properties of these edge states. We do that using two complementary approaches: the study of the so-called indirect exchange coupling [9,26,27], originally proposed by Ruderman, Kittel, Kasuya, Yosida (RKKY) [28-30], and the study of the emergent magnetism generated by Coulomb repulsion $[7,8,10,11,13]$. For the second, we use two different methods, DFT calculations and mean-field Hubbard model calculations, which happen to give very similar results. In the case of pristine graphene, it is well known that both RKKY indirect exchange and the spin interactions between magnetic moments that spontaneously emerge due to Coulomb interactions comply with the rule that interactions between magnetic moments on the same sublattice are ferromagnetic, whereas moments on opposite sublattices interact antiferromagnetically [7-10,13,15,26,27].

The fact that indirect exchange for spins on the same (different) sublattice are correlated ferromagnetically (antiferromagnetically) is a direct consequence of the bipartite character of the lattice $[6,8,9]$. In order to see how this rule is modified due to the presence of the FG that breaks the bipartite lattice we consider the Hamiltonian $\mathcal{H}_{0}+\mathcal{V}$, where $\mathcal{H}_{0}$ describes the tight-binding model for the islands shown in Fig. 2) and $\mathcal{V}=J \sum_{\eta=1,2} \vec{m}_{\eta} \cdot \vec{S}_{\eta}$, describes two classical moments $\vec{m}_{1}$ and $\vec{m}_{2}$ that are exchange coupled to the local spin density $\vec{S}_{i}=\sum_{\sigma, \sigma^{\prime}} \frac{1}{2} c_{i \sigma}^{\dagger} c_{i \sigma^{\prime}} \vec{\tau}_{\sigma, \sigma^{\prime}}$ of the graphene electrons. Here, $\vec{\tau}_{\sigma, \sigma^{\prime}}$ are the Pauli matrices.

The indirect exchange interaction between the magnetic moments at sites 1 and 2 is given by [26]

$$
\mathcal{J}_{12}=J^{2} \chi_{12} \vec{m}_{1} \cdot \vec{m}_{2}
$$

where $\chi_{i j}$ is the nonlocal spin susceptibility function, defined as the variation of the spin density in site $i$ due to the application 
of a local Zeeman field $\vec{b}$ in site $j$ :

$$
\left\langle\vec{S}_{i}\right\rangle=\chi_{i j} \vec{b}_{j} .
$$

The nonlocal spin susceptibility can be obtained analytically for $2 \mathrm{D}$ graphene $[9,26]$, using linear response theory. For the systems considered here this is not possible and, following previous work [27], we compute the expectation value of the spin density $\left\langle\vec{S}_{i}\right\rangle$ using the exact eigenstates of $\mathcal{H}_{0}+\vec{b}_{1} \cdot \vec{S}_{1}$, where $\mathcal{H}_{0}$ is the tight-binding Hamiltonian of the island, and $\vec{b}_{1}$ is a local magnetic field acting on atom 1 only. By doing so, we obtain the entries $\chi_{i 1}$ of the susceptibility matrix. Repeating this procedure changing the location of the perturbed site we obtain the complete matrix. For a system with $N$ atoms, this requires $N$ diagonalizations, and for each of them, the computation of $N$ spin densities.

In Fig. 2(a) we plot the map of $\chi_{i j_{0}}$, that represents the change in spin density induced in the sample when we apply a local field in one of the edge atoms, labeled by $j_{0}$, for a structure with one FG per zigzag edge, so that there are four edge fragments. It is apparent that same fragment correlations are the largest and ferromagnetic, whereas interactions with the other fragments are antiferromagnetic and smaller. These results are in contrast with the indirect exchange interactions obtained for pristine graphene, which are determined entirely by the sublattice degree of freedom $[9,26,27]$. The fact that the coupling of one edge fragment with all the others is antiferromagnetic implies that there will be some sort of spin frustration. This follows from the fact that in a pristine ribbon, the correlation between opposite edges is antiferromagnetic. Since the fluoranthene group also induces antiferromagnetic correlations within the edge, the total system consists of localized spins, which are all correlated antiferromagnetically. In the case of the figure, it is worth noticing that the spin correlation with the moments located at the opposite edge are weaker for the moments located in front, compared with those in the diagonal. This anticipates the magnetic ground state that arises from the effective spin Hamiltonian.

We now discuss the inhomogeneous magnetic order that would arise if we had a set of classical magnetic moments $\vec{m}_{i}$ at each site of the lattice, interacting with the indirect exchange interaction mediated by the carriers in the graphene nanoisland, governed by the Hamiltonian $\mathcal{H}=\sum_{i j} \mathcal{J}_{i, j} \vec{m}_{i}$. $\vec{m}_{j}$. For that matter we use the following method devised by Anderson [31]. We treat the magnetic moments in the meanfield approximation, which permits us to write the free energy of the system as:

$$
\mathcal{F}\left(m_{i}\right)=\sum_{i}\left(\frac{\vec{m}_{i}^{2}}{2 \chi_{0}}-\vec{m}_{i} \vec{b}_{i}\right),
$$

where $\vec{b}_{i}=\sum_{j} \mathcal{J}_{i j} \vec{m}_{j}$ is the effective field created by the interaction between the spins $m_{i}$, treated at the mean-field level and $\chi_{0}=\frac{\left(g \mu_{B}\right)^{2} S(S+1)}{3 k_{B} T}$ is the paramagnetic Curie susceptibility of the local moments. In equilibrium, we have $0=\frac{\delta \mathcal{F}}{\delta m_{i}}$ that leads to $\sum_{j} \mathcal{J}_{i j} m_{j}=\frac{m_{i}}{\chi_{0}}$. This equation is always satisfied by the disordered nonmagnetic solution, with $m_{i}=0$. However, it could also be satisfied by the eigenvectors of the interaction matrix, provided that their eigenvalues $\lambda$ satisfy $\lambda=\chi_{0}^{-1}$. At very large temperature $\chi^{-1}$ will be larger than the maximal $\lambda_{\max }$. However, as the temperature is reduced below $T_{c}$ such that $\lambda_{\max }=\chi_{0}\left(T_{c}\right)^{-1}$ the system will order, and the magnetic order will be given by the eigenstate $m_{i}^{\max }$ corresponding to $\lambda_{\max }$.

We apply this procedure to compute the magnetization map to three structures: a pristine graphene ribbon [Fig. 2(c)], as well as two functionalized graphene ribbons with different lengths $L$ [Figs. 2(b), 2(d)]. In all cases magnetism emerges at the zigzag edge atoms, the bulk sites remain nonmagnetic. In the pristine case, we find that both zigzag edges would order ferromagnetically, with opposite magnetizations, expected from the standard RKKY in pristine graphene, and given that all atoms in a given edge belong to the same sublattice. In the functionalized ribbons we also find ferromagnetic correlations between atoms on the same edge, provided that they are not separated by the FG. The main novelty is the antiferromagnetic correlations between magnetic moments on the same edge, separated by the FG. The interedge correlations are also different than in the pristine case for the shorter ribbon, ferromagnetic, whereas in the longer one they are antiferromagnetic. The interedge correlations reflect the competition between the antiferromagnetic couplings with a given edge fragment and the other three.

We now address the question of whether magnetic moments can arise in the functionalized zigzag edges due to Coulomb interactions, as it was predicted to happen in the case of pristine edges [7,8,10-13]. There, local moments with ferromagnetic correlations are expected on account of the Hund's exchange between the degenerate $E=0$ modes whose wave functions overlap along the edge. The presence of the FG functionalization changes the situation (see Fig. 1), giving rise to edge states that are linear combination of states localized at both sides of the FG. In this scenario, Coulomb repulsion is expected to result in antiferromagnetic interactions between the edge portions separated by the defect, and that is why interedge correlations in pristine zigzag edges are antiferromagnetic as well.

We discuss first the results obtained with DFT calculations. We consider the functionalized island of Fig. 3. The edge carbon atoms are passivated with hydrogen. The calculation is done with the quantum chemistry code GAUSSIAN 09 [32].

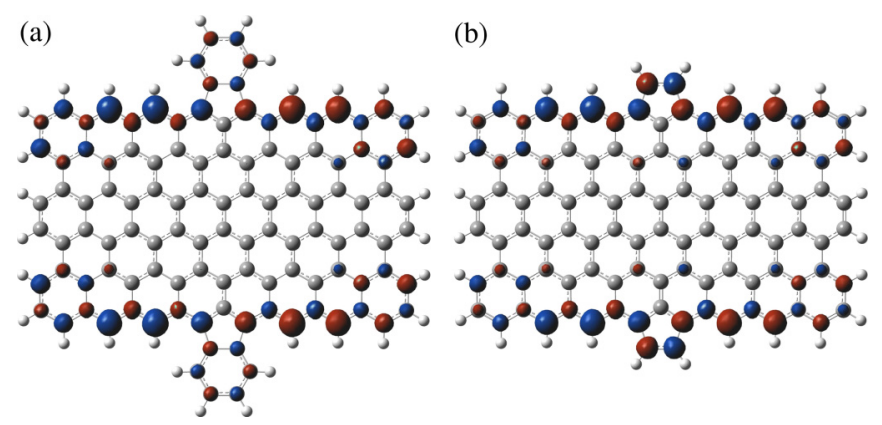

FIG. 3. Isocontours of the spin density obtained with DFT calculations, for two different systems (a) and (b), showing in both cases intraedge antiferromagnetic correlations. The color stands for the sign of the magnetic moment. Calculations were performed with CAM - B3LYP $/ 6-31 \mathrm{~g}(\mathrm{~d}, \mathrm{p})$, and the isosurfaces plotted correspond to isovalue 0.005 . 
The results shown here are obtained using the CAM-B3LYP functional with the basis set 6-31g(d,p) [17], yet similar results were also obtained with B3LYP and PBE0 functionals.

We computed three different spin configurations at the DFT level: zero spin, antiferromagnetic, and ferromagnetic. In all cases the molecular geometry was relaxed until forces were below $0.024 \mathrm{eV} / \mathrm{A}$ and $0.013 \mathrm{eV} / \mathrm{rad}$. Planar and distorted initial molecular geometries yielded fully equivalent results upon relaxation, which is not surprising since both graphene and the fluoranthene molecule are planar themselves. DFT yielded a magnetic ground state with all the functionals tried, namely: PBE0, B3LYP and CAM-B3LYP. Unpolarized solutions appeared at higher energies ranging from 0.3 (B3LYP) to $0.7 \mathrm{eV}$ (CAM-B3LYP). Figure 3(a) depicts the spin density for the antiferromagnetic state solution. The magnetization under the FG is depleted. The arrangements of the magnetic moments is different from the one obtained for pristine edges, and in line with those predicted by the RKKY interactions for this system. We find an antiferromagnetic coupling between the magnetic moments on the same edge that are separated by the FG, whereas ferromagnetic correlation between moments facing each other in opposite edges.

The fact that this peculiar arrangement is originated by the breakdown of the bipartite character of the lattice is confirmed by the results of the calculation shown in Fig. 3(b). There we consider a functionalization of a single pentagon, without the additional four carbon atoms that form the external hexagon of the FG. The magnetization profile obtained for this structure shares the same set of spin correlations. Therefore, it is the presence of the pentagon group the one responsible of the antiferromagnetic coupling between same-edge atoms separated by the defect. Interestingly, a pentagon group such as the one in in Fig. 3(b) could be formed by reconstruction of Klein ribbon edges [33] or carbon nanotube unzipping [34].

The emergent magnetism in zigzag edges can be described as well using the Hubbard model, given by

$$
\mathcal{H}=-\sum_{\langle i j\rangle, \sigma} t c_{i \alpha}^{\dagger} c_{j \alpha}+U \sum_{i} n_{i \uparrow} n_{i \downarrow} \equiv \mathcal{H}_{0}+\mathcal{H}_{U},
$$

where $n_{i \uparrow}=c_{i \uparrow}^{\dagger} c_{i \uparrow}$ denotes the occupation operator of site $i$ with spin $\uparrow$ along an arbitrary quantization axis. In the meanfield approximation the results (magnetic moment density, energy spectrum) are very similar to those obtained with DFT $[8,11]$. The model has the advantage of being computationally less expensive and it can also be treated going beyond mean-field interactions, that permits to go beyond the broken symmetry analysis of magnetism and study thereby dynamic spin fluctuations [35].

Here we first treated the model at the noncollinear meanfield approximation [24,25]. However, we always found collinear solutions for this system, with all the moments along a common axis that we take as the spin quantization axis. With that in mind, the mean-field Hamiltonian can be simplified into a collinear form:

$$
\left.\mathcal{H}^{\mathrm{MF}}=\mathcal{H}_{0}+U\left[\left\langle n_{i \uparrow}\right\rangle n_{i \downarrow}+n_{i \uparrow}\left\langle n_{i \downarrow}\right\rangle\right\rangle\right],
$$

where $\left\langle n_{i \uparrow}\right\rangle$ stand for the average of the occupation operator calculated within the ground state of the mean-field Hamiltonian (5). The mean-field Hamiltonian is a functional (a)

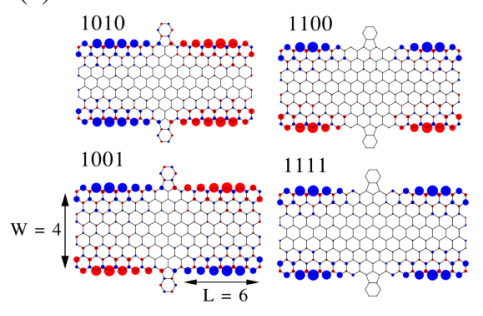

(c)

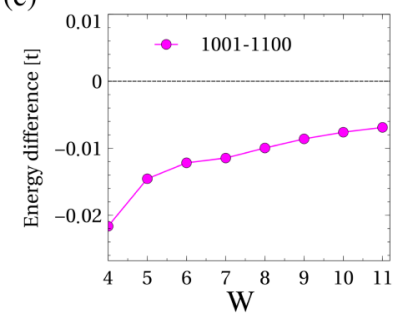

(b)

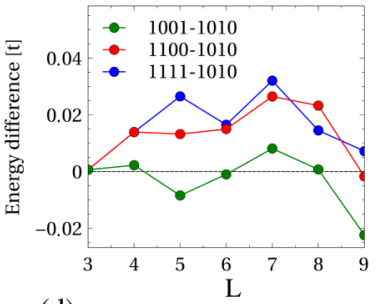

(d)

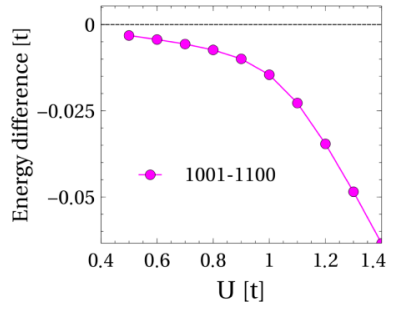

FIG. 4. (a) Magnetization profile of various self-consistent configurations obtained within the mean-field Hubbard model. Red and blue stand for the sign of the magnetic moment. The size of the symbols stands for the magnitude of the magnetic moment. (b) Evolution of the energy difference between the four configurations shown in panel (a) as a function of the size of the ribbon $L$. The lowest energy configurations are always the 1010 or the 1001 , the ones showing intraedge antiferromagnetic correlations. (c) shows the energy difference between 1001 and 1100 as the island becomes thicker for $L=5$, whereas (d) shows the energy difference as a function of $\mathrm{U}(W=L=5)$. Exponential extrapolation of (c) to $W=\infty$ gives a total energy difference of 0.007 [t], $19 \mathrm{meV}$ for $t=2.7 \mathrm{eV}$

of $\left\langle n_{i \sigma}\right\rangle$, which in turns depends on the eigenstates. This defines a self-consistent problem, which we solve by numerical iteration. In the following, unless stated otherwise, we take $U=t$.

Adequate choice of the initial condition for the procedure can result in the convergence of different solutions, that provide a local minima in the landscape of different mean-field solutions. We obtain several of these solutions for a series of graphene ribbons with one FG per edge, shown in Fig. 4. They all have spontaneous atomic magnetic moments at the zigzag edges, $m_{i}=\frac{\left\langle n_{i \uparrow}\right\rangle-\left\langle n_{i \downarrow}\right\rangle}{2}$ that order ferromagnetically in the edge fragments as long as they are not interrupted by a FG. The magnetization is depleted at the location of the defect. The magnitude of the magnetic moment away from the defects is the same than the one obtained for pristine edges.

The difference between the various self-consistent solutions, shown in Fig. 4(a), lies on relative magnetization orientation of the four ferromagnetic fragments. We label the magnetic orientation of a given edge fragment with respect to the spin quantization axis with either 0 or 1 . Using this notation, we can label the four possible distinguishable magnetic states of the structures with one defect per edge. The mean-field approximation permits us to compute their energies through the expression:

$$
E_{G}=\sum_{n} \epsilon_{n}-U \sum_{i}\left\langle n_{i \uparrow}\right\rangle\left\langle n_{i \downarrow}\right\rangle
$$


where $\epsilon_{n}$ stands for the eigenstates of the mean-field Hamiltonian and the sum runs over the occupied states only. We can compare the energies of the different magnetic configurations and infer the effective spin couplings between the magnetized edge fragments. In Fig. 4(b) we show the evolution of the ground-state energies as a function of the lateral dimension of the island, L. It is apparent that the dominant exchange coupling between different edge fragments is the intraedge antiferromagnetic coupling across a defect. For sufficiently short structures, the ground state displays ferromagnetic correlations between edge fragments located face to face. For structures with larger $L$ the ground state has antiferromagnetic correlations between opposite edge fragments. This, together with the analysis of the indirect exchange coupling, suggests that the effective couplings between all fragments are antiferromagnetic. For shorter structures the diagonal coupling outweighs the face-to-face interaction and the situation is reverted for larger ribbons. The energy differences between different magnetic configurations is in the range of $10^{-2} t \simeq$ $30 \mathrm{meV}$. We also checked the scaling of the intraedge exchange coupling as the two edges become farther apart [Fig. 4(c)], obtaining an asymptotic value of the intraedge exchange of $9.5 \mathrm{meV}$ for graphene. The intraedge AF configuration is the stable in a wide range of $U$, as shown in Fig. 4(d), with the exchange coupling growing with $U$.

We now study how the magnetic properties of the edges evolve as we scale up their size, keeping a similar density of defects. We thus consider longer structures with several FGs at the edge, in line with those reported by Ruffieux et al. [20]. In Fig. 5 we show the results of our mean-field calculations for an elongated ribbon with three defects located symmetrically at both edges, that define four zigzag edge fragments per edge. We find that the same rules that govern exchange in the presence of a single FG, still apply for larger structures, which is not entirely obvious given the delocalized nature of the edge states along the edge direction. Therefore, we conclude that the structures shown by Ruffieux et al. [20] host magnetic moments localized at the edges with antiferromagnetic correlations for moments separated by a FG group.

In summary, we have demonstrated that graphene ribbons with zigzag edges decorated with fluoranthene groups reported by Ruffieux et al. [20] host edge magnetic moments, very much like their pristine counterparts, but the rules that govern the spin interactions between different edge fragments are reversed compared to pristine edges. Thus, whereas in pristine ribbons magnetic moments on the same edge are always (a)

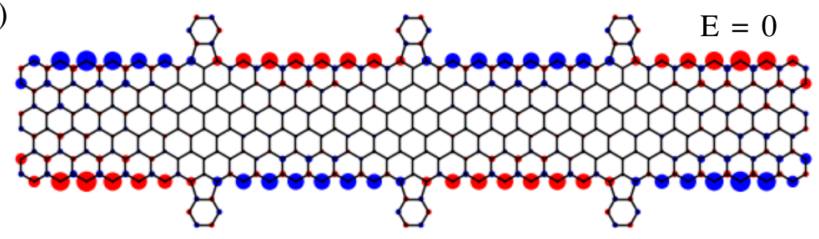

(b)

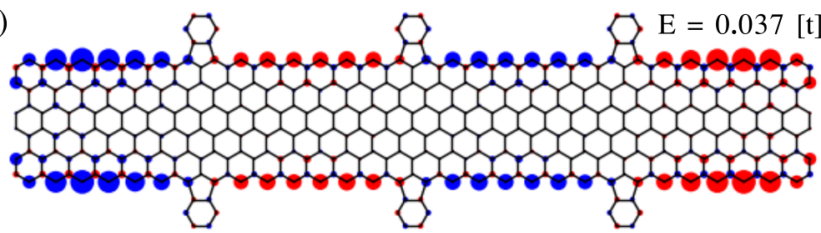

FIG. 5. (a) Magnetization profile for structure with several fluoranthene groups obtained within the mean-field Hubbard model $U=|t|$. Red and blue stand for the sign of the magnetic moment. The size of the symbols stands for the magnitude of $m_{i}$. (a) shows the ground-state configuration, whereas (b) an excited state.

ferromagnetically correlated [7,8,10,11,13], antiferromagnetic correlations are possible when moments in the same edge are separated by a single FG. The one-to-one relation between the sign of the spin correlations and the sublattice degree of freedom no longer applies, as the presence of a pentagon breaks down the bipartite character of the lattice. Thus, rather than being an unwanted defect on the ideal structures, the fluoranthene groups can used as a resource to nanoengineer spin-based quantum technologies based on nanographenes, and could also be used as platforms to study spin-liquid physics, spin ladders, Haldane chains, and other interesting and nontrivial quantum magnetism phenomena.

We acknowledge financial support by Marie-Curie-ITN 607904 SPINOGRAPH. J.F.R. acknowledges financial supported by MEC-Spain (FIS2013-47328-C2-2-P) and Generalitat Valenciana (ACOMP/2010/070), Prometeo. This work is funded by ERDF funds through the Portuguese Operational Program for Competitiveness and Internationalization COMPETE 2020, and National Funds through FCT, The Portuguese Foundation for Science and Technology, under the project "PTDC/FIS-NAN/4662/2014" (016656), CICECOAveiro Institute of Materials, POCI-01-0145-FEDER-007679 (FCT Ref. UID /CTM /50011/2013) and ON2 (NORTE07-0162-FEDER-000086) J.L.L. thanks the hospitality of the Departamento de Fisica Aplicada at the Universidad de Alicante.
[1] V. Meunier, A. G. Souza Filho, E. B. Barros, and M. S. Dresselhaus, Physical properties of low-dimensional $s p^{2}$ carbon nanostructures, Rev. Mod. Phys. 88, 025005 (2016).

[2] Bill Sutherland, Localization of electronic wave functions due to local topology, Phys. Rev. B 34, 5208 (1986).

[3] Gerardo G. Naumis, Internal mobility edge in doped graphene: Frustration in a renormalized lattice, Phys. Rev. B 76, 153403 (2007).
[4] Vitor M. Pereira, J. M. B. Lopes Dos Santos, and A. H. Castro Neto, Modeling disorder in graphene, Phys. Rev. B 77, 115109 (2008).

[5] M. Inui, S. A. Trugman, and Elihu Abrahams, Unusual properties of midband states in systems with off-diagonal disorder, Phys. Rev. B 49, 3190 (1994).

[6] Elliott H. Lieb, Two Theorems on the Hubbard Model, Phys. Rev. Lett. 62, 1201 (1989). 
[7] Mitsutaka Fujita, Katsunori Wakabayashi, Kyoko Nakada, and Koichi Kusakabe, Peculiar localized state at zigzag graphite edge, J. Phys. Soc. Jpn. 65, 1920 (1996).

[8] Joaquín Fernández-Rossier and J.-J. Palacios, Magnetism in Graphene Nanoislands, Phys. Rev. Lett. 99, 177204 (2007).

[9] Saeed Saremi, Rkky in half-filled bipartite lattices: Graphene as an example, Phys. Rev. B 76, 184430 (2007).

[10] Young-Woo Son, Marvin L. Cohen, and Steven G. Louie, Half-metallic graphene nanoribbons, Nature (London) 444, 347 (2006).

[11] Joaquín Fernández-Rossier, Prediction of hidden multiferroic order in graphene zigzag ribbons, Phys. Rev. B 77, 075430 (2008).

[12] J. Jung, T. Pereg-Barnea, and A. H. MacDonald, Theory of Interedge Superexchange in Zigzag Edge Magnetism, Phys. Rev. Lett. 102, 227205 (2009).

[13] Oleg V. Yazyev, Emergence of magnetism in graphene materials and nanostructures, Rep. Prog. Phys. 73, 056501 (2010).

[14] Oleg V. Yazyev and Lothar Helm, Defect-induced magnetism in graphene, Phys. Rev. B 75, 125408 (2007).

[15] J. J. Palacios, Joaquin Fernández-Rossier, and L. Brey, Vacancyinduced magnetism in graphene and graphene ribbons, Phys. Rev. B 77, 195428 (2008).

[16] Yasushi Morita, Shuichi Suzuki, Kazunobu Sato, and Takeji Takui, Synthetic organic spin chemistry for structurally welldefined open-shell graphene fragments, Nature Chem. 3, 197 (2011).

[17] Manuel Melle-Franco, Uthrene, a radically new molecule?, Chem. Commun. 51, 5387 (2015).

[18] Chenggang Tao, Liying Jiao, Oleg V. Yazyev, Yen-Chia Chen, Juanjuan Feng, Xiaowei Zhang, Rodrigo B. Capaz, James M. Tour, Alex Zettl, and Steven G. Louie, Spatially resolving edge states of chiral graphene nanoribbons, Nature Phys. 7, 616 (2011).

[19] Shiyong Wang, Leopold Talirz, Carlo A. Pignedoli, Xinliang Feng, Klaus Müllen, Roman Fasel, and Pascal Ruffieux, Nat. Commun. 7, 11507 (2016).

[20] Pascal Ruffieux, Shiyong Wang Wang, Bo Yang, Carlos Sánchez-Sánchez, Jia Liu, Thomas Dienel, Leopold Talirz, Prashant Shinde, Carlo A. Pignedoli, Daniele Passerone, Tim Dumslaff, Xinliang Feng, Klaus Muellen, and Roman Fasel, On-surface synthesis of graphene nanoribbons with zigzag edge topology, Nature (London) 531, 489 (2016).

[21] Héctor González-Herrero, José M Gómez-Rodríguez, Pierre Mallet, Mohamed Moaied, Juan José Palacios, Carlos Salgado, Miguel M. Ugeda, Jean-Yves Veuillen, Félix Yndurain, and Iván Brihuega, Atomic-scale control of graphene magnetism by using hydrogen atoms, Science 352, 437 (2016).

[22] María P López-Sancho, Fernando de Juan, and María AH Vozmediano, Magnetic moments in the presence of topological defects in graphene, Phys. Rev. B 79, 075413 (2009).
[23] Simone S. Alexandre, A. D. Lúcio, A. H. Castro Neto, and R. W. Nunes, Correlated magnetic states in extended one-dimensional defects in graphene, Nano Lett. 12, 5097 (2012).

[24] J. L. Lado and J. Fernández-Rossier, Magnetic Edge Anisotropy in Graphenelike Honeycomb Crystals, Phys. Rev. Lett. 113, 027203 (2014).

[25] J. L. Lado and J. Fernández-Rossier, Noncollinear magnetic phases and edge states in graphene quantum hall bars, Phys. Rev. B 90, 165429 (2014).

[26] L. Brey, H. A. Fertig, and S. Das Sarma, Diluted Graphene Antiferromagnet, Phys. Rev. Lett. 99, 116802 (2007).

[27] Annica M. Black-Schaffer, Rkky coupling in graphene, Phys. Rev. B 81, 205416 (2010).

[28] Melvin A. Ruderman and Charles Kittel, Indirect exchange coupling of nuclear magnetic moments by conduction electrons, Phys. Rev. 96, 99 (1954).

[29] Tadao Kasuya, A theory of metallic ferro-and antiferromagnetism on zener's model, Prog. Theor. Phys. 16, 45 (1956).

[30] Kei Yosida, Magnetic properties of $\mathrm{Cu}-\mathrm{Mn}$ alloys, Phys. Rev. 106, 893 (1957).

[31] Philip Warren Anderson, Localisation theory and the $\mathrm{Cu}-\mathrm{Mn}$ problem: Spin glasses, Mater. Res. Bull. 5, 549 (1970).

[32] M. J. Frisch, G. W. Trucks, H. B. Schlegel, G. E. Scuseria, M. A. Robb, J. R. Cheeseman, G. Scalmani, V. Barone, B. Mennucci, G. A. Petersson, H. Nakatsuji, M. Caricato, X. Li, H. P. Hratchian, A. F. Izmaylov, J. Bloino, G. Zheng, J. L. Sonnenberg, M. Hada, M. Ehara, K. Toyota, R. Fukuda, J. Hasegawa, M. Ishida, T. Nakajima, Y. Honda, O. Kitao, H. Nakai, T. Vreven, J. A. Montgomery, J. E. Peralta, F. Ogliaro, M. Bearpark, J. J. Heyd, E. Brothers, K. N. Kudin, V. N. Staroverov, R. Kobayashi, J. Normand, K. Raghavachari, A. Rendell, J. C. Burant, S. S. Iyengar, J. Tomasi, M. Cossi, N. Rega, J. M. Millam, M. Klene, J. E. Knox, J. B. Cross, V. Bakken, C. Adamo, J. Jaramillo, R. Gomperts, R. E. Stratmann, O. Yazyev, A. J. Austin, R. Cammi, C. Pomelli, J. W. Ochterski, R. L. Martin, K. Morokuma, V. G. Zakrzewski, G. A. Voth, P. Salvador, J. J. Dannenberg, S. Dapprich, A. D. Daniels, Farkas, J. B. Foresman, J. V. Ortiz, J. Cioslowski, and D. J. Fox, GAUSSIAN 09, Revision B.01, 2009.

[33] Kuang He, Alex W. Robertson, Sungwoo Lee, Euijoon Yoon, Gun-Do Lee, and Jamie H. Warner, Extended klein edges in graphene, ACS nano 8, 12272 (2014).

[34] Philipp Wagner, Viktoria V. Ivanovskaya, Manuel Melle-Franco, Bernard Humbert, Jean-Joseph Adjizian, Patrick R. Briddon, and Christopher P. Ewels, Stable hydrogenated graphene edge types: Normal and reconstructed klein edges, Phys. Rev. B 88, 094106 (2013).

[35] N. M. R. Peres, M. A. N. Araújo, and Daniel Bozi, Phase diagram and magnetic collective excitations of the hubbard model for graphene sheets and layers, Phys. Rev. B 70, 195122 (2004). 